\title{
INFORMES
}

\section{LA REUNIÓN PARALELA SOBRE POBLACIÓN Y DESARROLLO EN AMÉRICA LATINA (MEXICO, 1977): HACIA UN CONSENSO LATINOAMERICANO}

\author{
E. R. WEISS-ALTANER
}

Universidad de Québec en Montreal y Centro de Estudios en Población, Buenos Aires

\section{La Reunión Paralela}

Entre el 4 y el 6 de agosto de 1977, poco antes de inaugurarse en la ciudad de México la XVIII Conferencia General de la Unión Internacional para el Estudio Científico de la Población, un importante grupo de investigadores latinoamericanos celebró en esa metrópoli la Reunión Paralela sobre Población y Desarrollo en América Latina. Aunque ésta no admitía parangón, en cuanto al número de temas y ponencias, con la Conferencia Regional Latinoamericana de Población de 1970, las ponencias de la Reunión Paralela y los comentarios que suscitaron permitieron entrever el consenso que ha surgido, desde 1970, entre los especialistas latinoamericanos acerca de las relaciones entre población y desarrollo. En las páginas siguientes se reseñarán las ponencias presentadas y se intercalarán algunas obsèrvaciones del autor.

\section{Las ponencias}

En los tres días de la Reunión Paralela se celebraron cinco sesiones. En la primera se configuró a grandes rasgos el binomio población y desarrollo, mientras que en la sesión siguiente se examinó el grado de satisfacción que reciben las necesidades básicas de enseñanza, alimentación, salud y vivienda. El segundo día comenzó con ponencias sobre la influencia de programas de planificación familiar en la demografía de la región, y culminó con la sesión sobre el crecimiento demográfico y los mercados de trabajo rurales y urbanos. En la sesión sobre las políticas de población como instrumento de desarrollo en América Latina se puso fin a la Reunión Paralela. 
Waldomiro Pecht, organizador de la sesión inicial, destacó el progreso registrado en América Latina por muchos indicadores económicos y sociales durante los años 1960-1975, de rápida expansión demográfica. Aunque el avance fue limitado en algunos conceptos, como la salud, el comportamiento global de la región desmintió los pronósticos de un estancamiento de la producción y bienestar por habitante como consecuencia de su crecimiento demográfico acelerado. Pecht señaló que el progreso registrado en los indicadores globales oculta la experiencia desfavorable, en punto a ingreso y bienestar, de la mayoría de las familias latinoamericanas.

Las desigualdades de ingreso, propiedad y poder constituyeron el telón de fondo de casi todas las ponencias, comentarios e intervenciones del público. José Alberto Magno de Carvalho y Charles Wood, utilizando la esperanza de vida al nacer como barómetro del efecto conjunto de los determinantes de la salud, y basándose especialmente en el Censo de 1970, demostraron claramente cómo la aguda desigualdad de ingresos por habitante en Brasil -entre regiones, grupos de ingreso y lugar de residencia rural o urbano- coincidía con una desigualdad en las tasas de esperanza de vida al nacer. Angel Fucaraccio encuadró esta disparidad dentro de la incorporación de la región latinoamericana, como productor industrial, en la nueva división internacional del trabajo que se perfila en el interior de la red capitalista desde 1960. La industrialización acelerada de algunos países latinoamericanos se manifiesta en condiciones de dependencia financiera, comercial y tecnológica que reducen a signos negativos el saldo neto de las corrientes económicas que fluyen entre la región y las potencias capitalistas, notablemente los Estados Unidos.

En realidad, la doble expansión - socioeconómica y demográfica- no debe sorprender, pues distintos grupos sociales controlan la creación de bienes y de personas. Esos grupos persiguen objetivos diferentes y a veces antagónicos, de modo que no cotejan ni armonizan sus planes de expansión. La reproducción de la fuerza de trabajo se da en hogares, es decir, bajo condiciones de extrema descentralización y de "fácil entrada a la producción". El control que ejercen los hogares latinoamericanos sobre los medios de producción y la circulación de mercancías es, para la mayoría de ellos, escaso y precario. Esto los margina de las decisiones sobre cuánto, cómo, qué y dónde producir los bienes cuyo aumento registran los indicadores de producto, ahorro, inversión y consumo nacionales. Una fracción reducida de hogares controla las empresas que concentran la producción e inversión. La creación de bienes y de personas no se vinculan al nivel decisional sino más bien por medio del trabajo no remunerado que fluye de la mayoria de las familias latinoamericanas a las empresas activas en la región. Aun los fondos procedentes del exterior son costeados, a largo plazo, por el trabajo local, según demuestran los estudios sobre el costo real para América Latina del financiamiento externo y de las importaciones de tecnología.

Vilmar Faria, cuya ponencia cerró la primera sesión, destacó el cambio de paradigmas que se ha producido, en los últimos años, entre los estudiosos de población latinoamericanos. La crítica a la "sociología de la modernización" ha dado lugar a un marco teórico que no enfoca la 
reproducción de individuos vistos como entes independientes unos de otros, sino que interpreta la práctica demográfica de personas físicas como parte de un proceso de reproducción de la sociedad y de sus contradicciones. Faria subrayó la necesidad de pasar de la crítica del paradigma funcionalista anterior y comenzar a trabajar intensivamente para resolver los problemas teóricos y empíricos que plantea el nuevo paradigma. Uno podría aventurarse y ubicar el comienzo de la transición a la que alude Faria en 1970, fecha de la Conferencia Regional Latinoamericana de Población - que dio expresión a los paradigmas entonces en cursoy poco antes de la serie de coloquios y seminarios auspiciados por la Comisión de Población y Desarrollo del Consejo Latinoamericano de Ciencias Sociales (CLACSO), en que se fraguó el nuevo consenso.

En la tarde del primer día, la Reunión desglosó el nexo entre población y desarrollo en cuatro vínculos: las necesidades básicas de educación, alimentación, salud y vivienda. Hildebrando Araica, organizador de la segunda sesión, advirtió la falta de información al respecto y la necesidad de relacionar esas cuatro variables no solamente con el crecimiento global de la población sino con los movimientos por grupos etarios, lugar de residencia y así sucesivamente. En su ponencia, Jorge Padua registró el progreso que denotaron, entre 1960 y 1970 para toda la América Latina, la alfabetización, la población atendida por la enseñanza primaria, secundaria y universitaria; el gasto total educacional; y el porcentaje del producto y presupuesto nacionales destinados a la educación. Por desgracia, altos índices de ausentismo, deserción, retraso y fracaso estudiantiles e ineficiencia administrativa acompañaron a esos adelantos. Padua observó que, si bien el sistema educacional ayuda a reproducir las desigualdades en la formación que sustentan las divisiones de clase, para algunos grupos la educación formal puede servir para escalar una mejor posición social. (El éxodo de profesionales de América Latina muestra que la educación universitaria ofrece además una movilidad social internacional.) No es difícil rechazar, junto con Padua, la "desescolarización" de la sociedad. No se obtendrá la fuerza de trabajo mejor formada que necesita América Latina sino creando una buena y eficaz infraestructura educacional, cuya pedagogía y organización flexibles permitan además rescatar adultos y adolescentes ya activos en la vida económica pero rezagados en cuanto a la formación que deberían alcanzar.

La doctora Susana Icaza señaló las numerosas deficiencias en la producción, transporte, comercialización y utilización de alimentos, que contribuyen a la pésima nutrición de los pueblos de la región. La autora sugirió ciertos caminos técnicos para aumentar la capacidad productiva del sector agrícola, como el mejoramiento genético de plantas y animales; mayor contenido nutritivo de los alimentos; almacenamiento y comercialización más eficientes; y 'más personal capacitado en las distintas etapas que recorre el alimento desde el campo de cultivo hasta el consumidor final. Estas sugerencias y todas las que revisten un carácter estrictamente técnico son importantes; deben acogerse y continuarse. Al mismo tiempo, comentaron los doctores Elza Berquó y Hugo Behm Rosas, no cabe ilusionarse y creer que existen soluciones técnicas a problemas sociales. Cuando los vínculos entre las personas son lazos mercan- 
tiles y capitalistas, las innovaciones técnicas no surgen ni se difunden en primer lugar para satisfacer las necesidades básicas sino para adelantar las estrategias de unidades productivas individuales que se encuentran en distinto grado de competencia entre sí. No obstante, aun con transformaciones políticas fundamentales, el acelerado crecimiento demográfico plantea un problema de producción y distribución de alimentos cuya solución será larga y costosa, especialmente si el cambio político crea desorganización y descapitalización en el agro.

En su ponencia, el doctor Hugo Behm Rosas subrayó que, si bien el factor demográfico agrava el problema de salud, la principal contradicción se da entre la disponibilidad reducida de servicios de salud y la miseria, que genera una gama de necesidades de salud. Ambos polos de la contradicción expresan las divisiones de clase en América Latina. Por esta misma razón, según Luis Ratinoff, la "solución" del problema habitacional ha sido la siguiente: una alta tasa de ocupación de las estructuras, el no reemplazo de gran parte de las viviendas marginales e insalubres y el mantenimiento de una demanda insatisfecha que nutre los altos precios del núcleo edificado. Las actuales técnicas de construcción imponen un costo enorme a cualquier solución verdaderamente satisfactoria. Por eso y las razones políticas. de sobra conocidas, la explosión demográfica no recibirá una respuesta habitacional adecuada, señaló Ratinoff, añadiendo que los ya bajos niveles de vivienda por habitante probablemente no descenderán muchó más de aquí al año 2000.

La doctora Erica Taucher, organizadora de la sesión sobre el efecto de la planificación familiar en América Latina, ubicó a esos programas en una perspectiva que ella misma calificó de "ecléctica", y que ve en el crecimento poblacional una traba al desarrollo pero reconoce que la fecundidad sólo disminuirá si las familias con alta fecundidad logran alcanzar mejores niveles de educación, trabajo y salud. La doctora trató de relacionar algunos índices de la intensidad de los programas de planificación familiar. en distintos países de la región, con indicadores de natalidad, mortalidad infantil, esperanza de vida al nacer, producto interno bruto por habitante y el porcentaie de la población escolar que recibe enseñanza primaria. Las asociaciones cuantitativas se revelaron inconcluyentes, en parte por deficiencias estadísticas y el número reducido de observaciones. El nivel tan agregado de las variables en cuestión posiblemente tamhién haya disimulado los efectos de los programas de planificación familiar.

Tres ponencias investigaron el papel de los programas de planificación familiar, aue cobran importancia alrededor de 1965, en los descensos aue registró la fecundidad después de 1960 en Centroamérica y $\mathrm{Pa}$ namá (María L. García de Aybar y José Carvaial), Colombia (Juan B. Londoño) y la República Dominicana (Aníbal Faúndez). De estos trabajos, el primero exhibió un criterio más equilibrado sobre el punto en estudio: los programas de planificación familiar contribuyeron secundariamente a una transición de la fecundidad que ya había comenzado y que se observaba también entre grupos fuera del alcance de los programas de planificación familiar durante los años 1960-1975. García de Aybar y Carvajal notaron además que el efecto positivo del control de la fe- 
cundidad sobre la salud materna se disvirtuaba por la pésima condición de vida de tantas familias. Faúndez y Londoño reconcieron primacía a la planificarión familiar en el descenso de la fecundidad en los países que estudiaron, sin presentar con todo pruebas empíricas para descartar la influencia de otros factores. Sin embargo, un reciente artículo (citado por Londoño con referencia a otro punto) concluyó que el descenso en la fecundidad colombiana se debió, en los años sesenta, principalmente a la postergación de la nupcialidad, a métodos tradicionales de anticoncepción (como el coito interrumpido y el método del ritmo) y al aborto. ${ }^{1}$ George Robetrs, invitado a comentar la ponencia de Faúndez sobre la República Dominicana, destacó la dudosa confiabilidad de las respuestas a encuestas sobre el conocimento y uso de medios anticonceptivos, y la gran dificultad de aislar el efecto de los programas de planificación familiar en las reducciones en la fecundidad durante épocas de transformación general de la sociedad. Agustín Porras cerró la sesión con una ponencia en la que pasó revista a varias definiciones de la noción de "nacimientos evitados" e índices de la misma.

La difusión de conocimentos técnicos sobre la regulación de la fecundidad puede fácilmente acompañarse de intentos de alterar las opiniones y pautas valorativas del público. Algunos han criticado con severidad este aspecto de los programas de planificación familiar en América Latina, pues presenta a la explosión demográfica como la causa del atraso material del continente y plantea soluciones primordialmente demográficas a las insuficiencias sociales. Se trata de una crítica, merecida en muchos casos. que no debe hacer olvidar lo positivo de los conocimientos de planificación familiar, aue aumentan el ámbito decisional del individuo, en particular de la mujer. Por lo demás, toda actividad humana, por ser un comportamiento social, se inscribe en alguna ideología, o estructura valorativa. Cabe imaginar aue un estado socialista -es decir, un régimen que, en su práctica cotidiana, efectivamente trata de superar las divisiones de clase a largo plazo - procure convencer a la población para aue limite su ritmo reproductivo, porque la situación concreta del país no le permite satisfacer las necesidades de los incrementos en la población. También se puede vislumbrar que ese hipotético estado socialista considere los niveles de fecundidad como demasiado bajos, en comparación con las proyecciones de la demanda de mano de obra. Esto podría aumentar la presión sobre los fondos de inversión potenciales, al exigir la mecanización de muchas tareas productivas.

Alfredo Lattes, organizador de la cuarta sesión - sobre las repercusiones del crecimiento demográfico en los mercados de trabajo rurales y urbanos--, la inauguró con un vistazo general de la evolución de la población económicamente activa $\mathrm{v}$ de los cambios observados en el tamaño, estructura por edades y distribución urbano-rural de la población latinoamericana. Luego bosauejó un modelo de oferta $v$ demanda de fuerza de trabajo para vertebrar los análisis de la relación entre la población (oferta de la fuerza de trabajo) y el desarrollo (demanda de fuerza de trabajo). A las sugerencias del autor podría añadirse la de

1 J. E. Potter. M. Ordóñez G. y A. R. Measham. 1976, "The Rapid Decline in Colombian Fertility", Population and Development Review 2 (3-4): 509-28. 
identificar los mercados laborales más importantes, que se entrelazan y permiten comprender las migraciones interregionales y el desempleo en distintos lugares.

En su ponencia, Guillermo Flichman señaló que el pođer de absorción de mano de obra que exhibiera el sector agrícola en épocas pasadas no resurgirá. Si bien el crecimiento demográfico y económico, tanto en la región como en el resto del mundo, impulsará la producción agrícola latinoamericana, los niveles más altos de producción se alcanzarán con técnicas que aumentan la productividad por hectárea pero que igualmente fortalecen la productividad por trabajador. La redistribución de tierras e insumos asociados, y la creación de una población rural de pequeños propietarios, podría reducir el éxodo del campo, mas sólo temporalmente. Cabe añadir a lo afirmado por Flichman que tal reforma redistribuiría más equitativamente la presión de emigrar del campo, en vez de eliminarla. De todos modos, la necesidad social de liberar recursos para la producción no agrícola conllevaría, en el mediano y largo plazos, una estrategia de aumentar la productividad del trabajador agrícola. Tal estrategia cobraría más premura todavía en el contexto igualitario que es premisa política de la reforma agraria real y efectiva que aquí se ha concebido. Nuevamente, aun con un régimen político sideralmente distante de los contemporáneos, disminuirá el poder de absorción de la agricultura.

Brígida García, Humberto Muñoz y' Orlandina de Oliveira presentaron un estudio detallado de las historias de vida de 23 trabajadores rurales que, entre 1950 y 1959 , se incorporaron por primera vez al mercado laboral de la capital mexicana como obreros no calificados de las industrias de bienes de producción. Sus resultados, si bien preliminares, plantean dudas sobre ciertas generalizaciones muy en boga en cuanto a la contribución de los emigrantes rurales al desempleo y la población "marginal" en las ciudades. Según indicó la doctora Zulma Recchini de Lattes, comentarista invitada para esta ponencia, las historias de vida son una táctica investigativa que puede resultar muy útil para escoger entre esas generalizaciones.

La mano de obra đel año 1985 ya está presente, señaló Eduardo Arriaga. Aun con una perspectiva algo más lejana, hasta el año 2000, el efecto de una fecundidad más baja en el tamaño de la mano de obra del año 2000 será limitado. La presión demográfica sobre los mercados laborales ahora y en los próximos veinte años resulta de los movimientos de población pretéritos. Por tanto, las medidas para paliar el subempleo y desempleo tendrán que ser económicas y políticas en el mediano plazo. Más aún, los países latinoamericanos inician una etapa de industrialización "pesada", basada en la producción de bienes de capital y no sólo de consumo, y eso con técnicas que absorben menos mano de obra por unidad de producto que cuando las mismas ramas de actividad dominaban la producción industrial de las potencias capitalistas de hoy. Arriaga no dudaba tanto de la capacidad latinoamericana de lograr niveles más altos de producción e ingreso por habitante pero sí de su capacidad de resolver el problema del desempleo y subempleo, es decir, de distribuir el mayor ingreso entre toda la población. 
Las migraciones internacionales siguen de actualidad en América Latina, aunque ya no son las corrientes intercontinentales de 1930 y antes, sino principalmente flujos intralatinoamericanos. Tanto es así que, según demuestra la ponencia de Juan María Carrón, existen hoy en día mercados laborales que traspasan las fronteras internacionales, como los de ParaguayBrasil y Bolivia-Paraguay-Argentina. La realidad descrita por Carrón nos sugiere el tema de la existencia de periferias dentro de la periferia capitalista. ¿Cuál es el flujo neto de ingreso entre los países latinoamericanos?, ¿cuál es la contribución de las corrientes migratorias interamericanas a ese flujo neto? Se dice que el desarrollo de las potencias capitalistas ha generado y reproduce el retraso relativo de América Latina. No es inconcebible que el desarrollo dispar de las economías latinoamericanas comience ya a reflejar además mecanismos de dominación entre esos estados.

La última sesión de la Reunión Paralela se dedicó a las políticas de población como instrumento de desarrollo en América Latina. Su organizador, Raúl Benítez Zenteno, señaló que la oposición del sector privado a la programación del desarrollo a largo plazo por el estado fue desapareciendo durante los años cincuenta. Con la observación, en los censos de 1960 , del grado de expansión demográfica que registraba el continente, y el ocaso temporal de las dictaduras militares, la planificación estatal, en sus esfuerzos por asegurar la sobrevivencia del capitalismo en la región, abarcó las metas demográficas y las interrelaciones socio-económico-demográficas. En su ponencia, Juan Carlos Elizaga recorrió algunos modelos econométricos usados en la programación estatal, advirtiendo la tendencia hacia modelos multisectoriales y hacia modelos en que el comportamiento demográfico se determina en forma endógena. Es importante señalar al respecto que la investigación desagregada de esas interrelaciones es una etapa absolutamente crucial para escoger las formas que deben adoptar las ecuaciones de un modelo econométrico nacional o regional.

Enrique Brito Velázquez cerró la quinta sesión pasando revista a las diferencias económicas y demográficas entre las entidades federativas de México. La asociación estrecha entre ambos grupos de diferencias regionales indujo al autor a plantear la necesidad de adecuar las metas de fecundidad y las estrategias de desarrollo. Por desgracia, las metas sugeridas por Brito no guardaban relación con lo que se puede esperar en realidad, a la luz de los conocimentos ya bastante avanzados sobre los determinantes de la fecundidad. La fecundidad - proceso descentralizado por excelencia- disminuiría a medida que cambie el sentido, o la racionalidad, que un número elevado de niños representa hoy para muchas familias. Tal transformación resulta en gran parte de las alteraciones en el contexto económico y social de esas familias, que es reflejo principalmente de las consecuencias de la inversión y la asignación de recursos hechas por los grupos dominantes de América Latina. ¿Qué nos permite prever que los intereses de esos grupos concordarán con los cambios económicos que elevarían a las familias pobres a niveles de vida más acomodados, donde el gasto de muchos recursos por hijo constituiría una estrategia de vida a la vez factible y racional desde el punto de 
vista de esas familias? ¿Qué nos permite pronosticarle a la América Latina un pasaje del capitalismo dependiente basado en la mano de obra barata y descalificada a una etapa, si bien todavía dependiente de las potencias capitalistas, basada en la mano de obra cara y calificada?

\section{Hacia un consenso latinoamericano}

A diferencia de la Conferencia Regional Latinoamericana de Población de 1970, la mayoría de los ponentes, los comentaristas invitados y el público que intervino reconocieron la importancia primordial de la desigual distribución del poder, propiedad e ingreso en el binomio población y desarrollo. Las reducciones en la fecundidad no se adelantaron como soluciones principales de las insuficiencias en las necesidades básicas. En realidad, los descensos en la fecundidad que ya se difunden en la región - como en Chile, Costa Rica y Colombia- no se han visto acompañados de mejoras a corto plazo en las condiciones de vida de todos los habitantes. La fecundidad y los otros elementos del cambio demográfico constituyen un factor que ha de incorporarse a la programación a largo plazo pero, con tales horizontes, los factores económicos y políticos también cobran una fluidez potencial que debe tenerse en cuenta.

La labor de los grupos constituyentes de la Comisión de Población y Desarrollo de CLACSO, especialmente los de migración y reproducción, ha surtido efecto. Desde 1972, esos grupos han criticado las teorías y metodologías de la sociología funcionalista que orientaba la investigación demográfica a la sazón y muy patentes en la Conferencia Regional Latinoamericana de Población de 1970. Sustituyen esas teorías un número variado de enfoques que van desde estructuralismos hasta perspectivas marxistas de diversa índole. La etapa de la crítica metodológica cede el paso a propuestas de investigación concreta en esa gama de enfoques, que destacan los conflictos sociales como determinante principal de los movimientos de población y del múltiple comportamiento demográfico que exhiben los diversos grupos socioeconómicos.

La implantación, en casi toda América Latina, de regímenes de fuerza militar hicieron quiméricos los proyectos de comienzos de los años sesenta, de una gran coalición social que produjera crecimiento económico y consumo adecuado para todos, y que mantuviera las libertades de prensa, docencia y asociación política. Sin duda alguna, el nuevo modelo de estado en Latinoamérica, con la represión y la diáspora intelectual que ha provocado, ha contribuido mucho al nuevo consenso entre los especialistas de población que se manifestó durante la Reunión Paralela de 1977. 